\title{
Transition rates and nuclear structure changes in mirror nuclei ${ }^{47} \mathrm{Cr}$ and ${ }^{47} \mathrm{~V}$
}

\author{
D. Tonev, ${ }^{1,2}$ P. Petkov, ${ }^{1,3}$ A. Dewald, ${ }^{1}$ T. Klug, ${ }^{1}$ P. von Brentano, ${ }^{1}$ \\ W. Andrejtscheff, ${ }^{3}$ S. M. Lenzi, ${ }^{4}$ D. R. Napoli, ${ }^{5}$ N. Marginean, ${ }^{5}$ F. \\ Brandolini,${ }^{4}$ C. A. Ur,${ }^{4}$ M. Axiotis,${ }^{5}$ P. G. Bizzeti,${ }^{6}$ and A. Bizzeti-Sona ${ }^{6}$ \\ 1 Institut für Kernphysik der Universität zu Köln, D-50937 Köln, Germany \\ 2 Faculty of Physics, University of Sofia, 1164 Sofia, Bulgaria \\ 3 Bulgarian Academy of Sciences, Institute for Nuclear \\ Research and Nuclear Energy, 1784 Sofia, Bulgaria \\ 4 Dipartimento di Fisica and INFN, Sezione di Padova, Padova, Italy \\ 5 INFN, Laboratori Nazionali di Legnaro, Legnaro, Italy \\ 6 Dipartimento di Fisica and INFN, Firenze, Italy
}

(Dated: October 26, 2018)

\begin{abstract}
Lifetime measurements in the mirror nuclei ${ }^{47} \mathrm{Cr}$ and ${ }^{47} \mathrm{~V}$ were performed by means of the Doppler-shift attenuation method using the multidetector array EUROBALL, in conjunction with the ancillary detectors ISIS and the Neutron Wall. The determined transition strengths in the yrast cascades are well described by full $p f$ shell model calculations.

PACS numbers: 21.10.Tg, 23.20.Lv, 27.40.+z
\end{abstract}

Keywords: Gamma rays; High spin; Lifetimes; Mirror symmetry 


\section{INTRODUCTION}

The investigation of mirror nuclei along the $N=Z$ line is of considerable interest since it addresses directly the charge symmetry of the nuclear forces and the role of the Coulomb effects on nuclear structure. The nuclei ${ }^{47} \mathrm{Cr}$ and ${ }^{47} \mathrm{~V}$ are among the heaviest mirror nuclei where high spin spectroscopy studies are presently accessible without employing radioactive beams. So far, they have been the subject of numerous experimental and theoretical studies. One impressive result is the establishment [1, 2] of the yrast cascades until the band termination state (with spin/parity $I^{\pi}=31 / 2^{-} \hbar$ for these $1 f_{7 / 2}$ nuclei) and the successful description of these structures by shell model (SM) calculations [3]. The comparison of the experimental excitation energies $E_{x}$ in the yrast cascades of ${ }^{47} \mathrm{Cr}$ and ${ }^{47} \mathrm{~V}$ shown in Fig. 1 yields the Coulomb energy differences (CED). Recently, the behavior of the CED $\left[E_{x}\left({ }^{47} \mathrm{Cr}\right)-E_{x}\left({ }^{47} \mathrm{~V}\right)\right]$ as a function of spin was presented as an evidence for nuclear structure (alignment of particles) and shape changes [1, , 1, 5, 6]. One of the aims of the present work is to verify if these changes are reflected by the corresponding $B(E 2)$ transition strengths in both nuclei. Another topic of interest is the comparison of the $B(E 2)$ 's with the predictions of the extensive full $p f$ shell model calculations [3]. As already mentioned, the SM calculations provide a good description of the level energies in the investigated nuclei. The preliminary data available [0, 8] for the subpicosecond lifetimes of interest in ${ }^{47} \mathrm{~V}$ determined the choice of our experimental tool which is the Doppler-shift attenuation method (DSAM).

\section{EXPERIMENTAL DETAILS}

Excited states in ${ }^{47} \mathrm{Cr}$ and ${ }^{47} \mathrm{~V}$ were populated using the $2 \alpha n$ and $2 \alpha p$ exit channels, respectively, of the reaction ${ }^{28} \mathrm{Si}+{ }^{28} \mathrm{Si}$. The beam, with an energy of $110 \mathrm{MeV}$, was delivered by the XTU Tandem accelerator of the Laboratori Nazionali di Legnaro. The target consisted of $0.85 \mathrm{mg} / \mathrm{cm}^{2} \mathrm{Si}$ (enriched to $99.9 \%$ in ${ }^{28} \mathrm{Si}$ ) evaporated on a $15 \mathrm{mg} / \mathrm{cm}^{2} \mathrm{Au}$ backing. The deexciting $\gamma$ rays were registered with the EUROBALL III array [9], using only cluster and clover Ge detectors for the present measurement. Charged particles were detected with the ISIS silicon ball (40 $\Delta E$ - E telescopes) 10 and neutrons with the Neu- 
tron Wall (50 large volume liquid scintillator detectors) [11]. Events were collected when at least two $\gamma$ rays in the Ge cluster or clover segments and one neutron identified in the Neutron Wall were in coincidence or at least three Ge detectors plus one signal in neutron detector fired in coincidence. Gain matching and efficiency calibration of the Ge detectors were performed using ${ }^{152} \mathrm{Eu}$ and ${ }^{56} \mathrm{Co}$ radioactive sources. A standard add-back correction for Compton scattering was applied. The data were sorted into coincidence $\gamma-\gamma$ matrices whereby the detection of charged particles and/or neutrons was required. Under the trigger conditions employed, the yield of ${ }^{47} \mathrm{~V}$ was estimated to be $8 \%$ of the total cross-section while that of ${ }^{47} \mathrm{Cr}$ was found to be about $0.25 \%$ only. Therefore the particle gates had to be used to select the reaction channels of interest out of equally strong or stronger competing exit channels.

For the purposes of a DSAM measurement, the information on the detection angles of the $\gamma$ rays is of primordial importance because the Doppler shifts of their energies depend on the angle between the direction of the recoil velocity and the direction of observation. Therefore the cluster and clover detectors of EUROBALL were grouped into rings corresponding to approximately the same polar angle with respect to the beam axis. Taking into account the segmented structure of these detectors leads to the formation of ten rings positioned at angles of about $72,81,99,107,122,129,137,145,155$, and $163^{\circ}$. In the case of ${ }^{47} \mathrm{~V}$, the statistics allowed to use (particle gated) $\gamma-\gamma$ matrices where the angular information was conserved on both axes of the matrix, i.e., the events represented the registration of two coincident $\gamma$ rays by the detectors of two particular rings. In principle, 100 matrices of that type could be constructed corresponding to all possible two-ring combinations. We have sorted 30 such matrices selected on the basis of the quality of the information provided by them. Because of the weak reaction channel leading to ${ }^{47} \mathrm{Cr}$, in this case only ten different matrices were sorted where one of the axes was associated with a specific detection angle while on the other axis every detector (ring) firing in coincidence was allowed. 


\section{DATA ANALYSIS}

For the Monte-Carlo (MC) simulation of the slowing-down histories of the recoils we used a modified [12, 13] version of the program DESASTOP [14] written by G.Winter. In this version, the time-evolution of the recoil velocities in the target and stopper is followed in three dimensions. The electronic stopping powers used were obtained from the Northcliffe and Schilling tables [15] with corrections for the atomic structure of the medium along the lines discussed in ref. [16]. As suggested in ref. [17], an empirical reduction of $f_{n}=0.7$ was applied to downscale the nuclear stopping power predicted by the theory of Lindhard, Scharff and Schiøtt [18]. According to the calculations performed, the mean velocity of the recoils when leaving the target was about $3.9 \%$ of the velocity of light, and they needed in average $1.2 \mathrm{ps}$ to come to rest. The evaporation of charged particles, which is of importance for the velocity distribution of light residual nuclei, was taken into account in the MC simulation and led to better fits of the spectra. The database of about 10000 velocity histories was additionally randomized with respect to the experimental setup by taking into account the exact position of the EUROBALL detectors and their finite size. Complementary details on our approach for Monte Carlo simulation can be found in refs. [12, 13, 19].

The differences in the acquired statistics for the two mirror nuclei and the nature of the sorted data were the reason for the application of two different approaches for data analysis. The strength of the ${ }^{47} \mathrm{~V}$ reaction channel made it possible to apply the newly developed procedure for analysis of coincidence DSAM data where the gate is set on the shifted portion of the line shape of a transition directly feeding the level of interest [20]. Within this approach, the timing quality of the gated line shape is improved compared to the case where the gate includes also fully stopped events because they do not bring lifetime information. Moreover, gating from above allows the elimination of the uncertainties related to the unobserved feeding of the level of interest which perturb singles measurements and coincidence measurements where the gate is set on a transition deexciting a level fed by the level of interest. We had to use the latter approach for ${ }^{47} \mathrm{Cr}$ because of the weakness of its yield. The details of the analysing procedure used are presented in refs. [12, 21]. We only mention here that within its framework it is possible to investigate the influence 
of the unobserved feeding on the derived lifetimes. Fits of line shapes obtained using both approaches are presented in Fig. 2 which illustrates also the quality of the data. An error propagation calculation was performed to take into account the effect of the background subtraction on the errors of the gated line shapes.

\section{RESULTS}

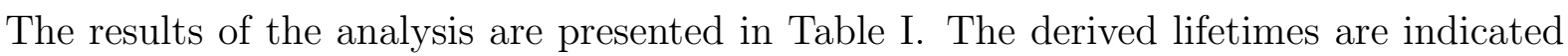
also in Fig. 1. In the case of ${ }^{47} \mathrm{~V}$, they were obtained from the analysis of gated spectra corresponding to 15 different two-ring combinations. The new lifetime values are in agreement with the data from earlier measurements. The lifetimes in ${ }^{47} \mathrm{Cr}$ are determined for the first time. For ${ }^{47} \mathrm{Cr}$, the analysed line shapes where obtained by gating on the $1157 \mathrm{keV}$ transition which does not show any shifted component. On top of the cascade (cf. Fig. 1) the statistics of the line shape of the $2006 \mathrm{keV}$ transition is too poor and prevents the precise determination of the lifetime of the $23 / 2^{-}$level. In the analysis, we have used the hypothesis that the time behavior of the unobserved feeding is the same as that of the known feeding. The intensity balance of the investigated levels in ${ }^{47} \mathrm{Cr}$ reveals that the fraction of the unobserved feeding is less than $10 \%$. Its influence, studied according to the procedure presented in ref. [12], leads to uncertainties within the statistical error bars, which makes the lifetime values derived more reliable. We estimate the uncertainty due to the imprecise knowledge of the stopping powers to $10 \%$ and include it in the final errors of the lifetimes given in Table [1. It should be noted that the derivation of lifetimes in ${ }^{47} \mathrm{Cr}$ and ${ }^{47} \mathrm{~V}$ in the same experiment makes the determination of the ratios of the corresponding transition strengths very precise since uncertainties related to the stopping powers nearly cancel. Moreover, our investigation represents the first determination of picosecond lifetimes of high-spin states in mirror nuclei from the $1 f_{7 / 2}$ shell associated with strong intraband transitions. 


\section{DISCUSSION}

Nuclei in the middle of the $1 f_{7 / 2}$ shell exhibit rotational properties at low and intermediate spins since the number of valence particles is sufficient to generate such collective effects. One very interesting result of the SM calculations [3] for this spin region in ${ }^{47} \mathrm{Cr}$ and ${ }^{47} \mathrm{~V}$ is the closeness of the predicted behavior of the $B(E 2)$ 's to the expectations of the rotational model [22]. We have derived from the $B(E 2)$ data transition quadrupole moments $Q_{t}$ using the equation

$$
B(E 2, I \rightarrow I-2)=\frac{5}{16 \pi}<I K 20 \mid I-2 K>^{2}\left(Q_{t}\right)^{2}
$$

The $Q_{t}$ values obtained in this way correspond to the intrinsic quadrupole moment $Q_{0}$ of the rotational model for a band with $K=3 / 2$. Using the relation 23

$$
Q_{0}=\frac{3}{\sqrt{5 \pi}} Z e R_{0}^{2} \beta\left(1+\frac{1}{8} \sqrt{\frac{5}{\pi}} \beta\right)
$$

we have also calculated the quadrupole deformation $\beta$ which can be associated with the measured transition strengths under the assumption of an approximate validity of the rotational model. The experimental $Q_{t}$ values are compared to the theoretical [3] ones in Table [ where the deduced values of $\beta$ are given too. The spin dependence of the $Q_{t}$ 's can be seen more easily in Fig. 3 where data available for other levels in ${ }^{47} \mathrm{~V}$ (taken from ref. [8]) and the full pf shell model predictions [24] are displayed. There is a very good agreement between the experiment and the theory. This indicates that the effective charges used $\left(e_{\pi}=1.5, e_{\nu}=0.5\right)$ are appropriate.

The information presented in Fig. 3 and Table 1 sheds light on several aspects of the nuclear structure of ${ }^{47} \mathrm{Cr}$ and ${ }^{47} \mathrm{~V}$. The behavior of the $Q_{t}$ 's determined in the present DSAM measurement in ${ }^{47} \mathrm{~V}$ shows a systematic decrease with increasing spin. The data for ${ }^{47} \mathrm{Cr}$ is less conclusive, but they exclude an increase of the $Q_{t}$ 's. The inset to Fig. 3 shows the Coulomb energy differences (CED) between the levels of the yrast cascades of ${ }^{47} \mathrm{Cr}$ and ${ }^{47} \mathrm{~V}$. Obviously, our $Q_{t}$ values characterize levels at which substantial changes in the CED occur. These changes were presented by Bentley et al. [1] as an evidence for changes in the underlying nuclear structure and the nuclear shape. The effect is explained by an alignment of a pair of protons in ${ }^{47} \mathrm{Cr}$ around spin $I=19 / 2 \hbar$ which leads to a reduction of their spatial 
correlations and thus of the Coulomb energy. This energy is not affected in ${ }^{47} \mathrm{~V}$ where two neutrons align. The present $Q_{t}$ values for ${ }^{47} \mathrm{~V}$ point at a sharper decrease of these quantities after the $I=19 / 2 \hbar$ level. This trend may be associated with the nuclear structure changes inferred earlier in Ref. [1] by observation of the behavior of the CED. However, the present data for ${ }^{47} \mathrm{Cr}$ does not allow to make the same conclusions and this point has to be clarified in the future by a more precise measurement of the lifetimes of the levels above the $I=19 / 2$ $\hbar$ one. Such a development with increasing spin has to be expected since the valence space of the $1 f_{7 / 2}$ nuclei considered is not very large. Its capability to generate collective effects gets exhausted when approaching the band-termination state whose angular momentum is built only from the contributions of individual nucleons. Since all valence protons align in both nuclei ${ }^{47} \mathrm{Cr}$ and ${ }^{47} \mathrm{~V}$, the $\mathrm{CED}$ close to the band termination state reach similar values to those at the lowest spins [4]. Recently, the behavior of the CED in several $f_{7 / 2}$ mirror nuclei has also been studied in the framework of the shell model [25]. The $A=47$ CED have been reproduced with good accuracy by including the change in nuclear radii along the yrast states together with a renormalization of Coulomb matrix elements in the pf shell. We note that there is a small disagreement between the experiment and the shell model calculations at $\operatorname{spin} I^{\pi}=19 / 2^{-}$(Fig. 3), which indicates that the shape changes are not fully satisfactory described by the model.

In a different theoretical framework, the cranked Nilsson-Strutinski calculations quoted in ref. [1] predict for ${ }^{47} \mathrm{Cr}$ a gradual change of the deformation from $\epsilon=0.21$ to about $\epsilon=$ -0.03 (oblate) at the $I^{\pi}=31 / 2^{-}$band-termination states. Our values for $\beta$ at the beginning

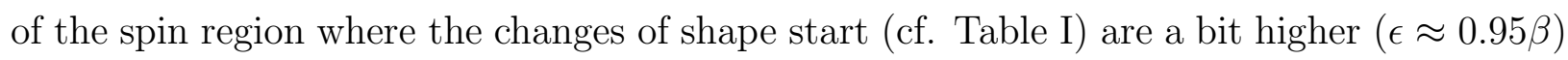
than these predictions. The agreement is even better if we reduce the deformation derived from the transition probabilities by a factor of about 1.1 to compare it with the deformation of the mean nucleon field (see e.g. ref. [26]). As indicated clearly by the data for ${ }^{47} \mathrm{~V}$, the deformation decreases with increasing spin. This evolution is a good example for an interplay between collective and microscopic degrees of freedom.

The nuclear forces are usually considered to be charge symmetric and one expects the same structural properties in mirror nuclei with differences caused only by the Coulomb interaction. The extent of the mirror symmetry can be already deduced from Fig. 11 which 
compares the level schemes of ${ }^{47} \mathrm{Cr}$ and ${ }^{47} \mathrm{~V}$. Our lifetime investigation shows that this symmetry is observed also in the transition probabilities. The small differences observed can be understood in terms of the extra proton in ${ }^{47} \mathrm{Cr}$. The comparison of the experimental $Q_{t}$ values in these mirror nuclei yields information on the differences in the wave functions of the corresponding states and the transition quadrupole moments. To disentangle these two factors is beyond the scope of the present work but may help further refinement of the theory.

\section{CONCLUSIONS}

To conclude, DSAM lifetime measurements were carried out with the multidetector array EUROBALL. The results of the analysis, partly achieved with a new precise procedure [20], provide valuable information on the transition strengths in the yrast cascades of the mirror

nuclei ${ }^{47} \mathrm{Cr}$ and ${ }^{47} \mathrm{~V}$. The behavior of the transition strengths with spin is well described by full $p f$ shell model calculations. In this way, a test of the isospin symmetry in mirror nuclei is performed on the basis of the determined $B(E 2)$ values.

\section{Acknowledgments}

Two of us (D.T. and P.P.) are grateful for the kind hospitality of the Cologne University. This work was funded by the BMBF under contracts No. 06 OK 862 I (0) and 06 OK 958 and by the European Commission through the Contract ERBFMCT980110 E. U. TMR Programme. The support of the Bulgarian National Research Foundation (BNRF) under contract $\mathrm{Ph} .801$ is also appreciated.

[1] M. A. Bentley et al., Phys. Lett. B 437, 243 (1998).

[2] J. A. Cameron et al., Phys. Rev. C 49, 1347 (1994).

[3] G. Martínes-Pinedo, A. P. Zuker, A. Poves, and E. Caurier, Phys. Rev. C 55, 187 (1997).

[4] M. A. Bentley et al., J. Phys. G 25, 599 (1999). 
[5] J. A. Cameron et al., Phys. Lett. B 319, 58 (1993).

[6] S. M. Lenzi et al., Phys. Rev. Lett. 87, 122501 (2001).

[7] J. A. Cameron et al., Phys. Rev. C 58, 808 (1998).

[8] F. Brandolini et al., Nucl. Phys. A 693, 517 (2001).

[9] J. Simpson, Z. Phys. A 358, 139 (1997).

[10] E. Farnea et al., Nucl. Phys. A 642, 347 (1998).

[11] Ö. Skeppstedt et al., Nucl. Instrum. Methods Phys. Res. A 421, 531 (1999).

[12] P. Petkov et al., Nucl. Phys. A 640, 293 (1998).

[13] P. Petkov, D. Tonev, J. Gableske, A. Dewald, T. Klemme, and P. von Brentano, Nucl. Instrum. Methods Phys. Res. A 431, 208 (1999).

[14] G. Winter, ZfK Rossendorf Report ZfK-497, 1983; G. Winter, Nucl. Instrum. Methods 214, $537(1983)$.

[15] L. C. Northcliffe and R. F. Schilling, Nucl. Data, Sect. 7, 233 (1970).

[16] J. F. Ziegler and J. P. Biersack, in Treatise on Heavy-Ion Science, edited by D. A. Bromley (Plenum Press, New York, 1985), Vol. 6, p. 95.

[17] J. Keinonen, in Capture Gamma-Ray Spectroscopy and Related Topics-1984, Proceedings of the Fifth International Symposium, Knoxville, TN, 1984, edited by S. Raman, AIP Conf. Proc. No. 125 (AIP, New York, 1985), p. 557.

[18] J. Lindhard, M. Scharff, and H. E. Schiøtt, K. Dan. Vidensk. Selsk. Mat. Fys. Medd. 33, 14 (1963).

[19] D. Tonev et al., Nucl. Instum. Methods Phys.Res. A (to be published).

[20] P. Petkov, D. Tonev, J. Gableske, A. Dewald and P. von Brentano, Nucl. Instrum. Methods Phys. Res. A 437, 274 (1999).

[21] G. Böhm, A. Dewald, P. Petkov and P. von Brentano, Nucl. Instrum. Methods Phys. Res. A 329, 248 (1993).

[22] A. Bohr and B. R. Mottelson, Nuclear Structure (Benjamin, New York, 1975), Vol. 2.

[23] A. Bohr and B. R. Mottelson, K. Dan. Vidensk. Selsk. Mat. Fys. Medd. 30,1 (1955)

[24] E. Caurier, computer code ANTOINE, CRN, Strasbourg, (1989).

[25] A. P. Zuker et al., nucl-th/0104048. 
[26] W. Nazarewicz, M. A. Riley, J. D. Garett, and J. Dudek, Nucl. Phys. A 512, 61 (1990). 
FIG. 1: Partial level schemes of ${ }^{47} \mathrm{Cr}$ and ${ }^{47} \mathrm{~V}$ from ref. [1] showing mainly the yrast cascades of the two nuclei. Lifetimes determined in the present work are displayed in framed boxes. Additional lifetime information from ref. [8] is also shown.

FIG. 2: Fits of line shapes of transitions in ${ }^{47} \mathrm{Cr}$ and ${ }^{47} \mathrm{~V}$ measured at different angles.

FIG. 3: Measured and theoretical transition quadrupole moments. The inset shows the behavior of the CED between ${ }^{47} \mathrm{Cr}$ and ${ }^{47} \mathrm{~V}$. The arrow indicates that the $Q_{t}$ value for the $23 / 2^{-}$level in ${ }^{47} \mathrm{Cr}$ is only a lower limit. 
TABLE I: Analysed transitions, derived lifetimes $\tau$, and transition quadrupole moments $Q_{t}$. The deformation $\beta$ determined using eq.2 is displayed in the last column. Branching ratios necessary for the derivation of $Q_{t}$ were taken from ref. [2]. Uncertainties of the experimental quantities are shown in brackets.

\begin{tabular}{|c|c|c|c|c|c|c|}
\hline Transition & $\begin{array}{l}\mathrm{E}_{\gamma} \\
k e V\end{array}$ & $\begin{array}{c}\tau \\
{[p s]}\end{array}$ & $\begin{array}{c}\tau_{\text {previous }} \\
{[p s]}\end{array}$ & $\begin{array}{l}Q_{t}^{\text {experiment }} \\
\text { efm }^{2}\end{array}$ & $\begin{array}{l}Q_{t}^{\text {theory }} \\
\text { efm } m^{2}\end{array}$ & $\beta$ \\
\hline \multicolumn{7}{|c|}{${ }^{47} \mathrm{Cr}$} \\
\hline $15 / 2^{-} \rightarrow 11 / 2^{-}$ & 1321 & $0.84(12)$ & - & $90(7)$ & 91 & $0.255(18)$ \\
\hline $19 / 2^{-} \rightarrow 15 / 2^{-}$ & 1485 & $0.44(6)$ & - & $88(6)$ & 73 & $0.248(16)$ \\
\hline $23 / 2^{-} \rightarrow 19 / 2^{-}$ & 1766 & $<0.64$ & - & $>44$ & 69 & $>0.127$ \\
\hline \multicolumn{7}{|c|}{${ }^{47} \mathrm{~V}$} \\
\hline $15 / 2^{-} \rightarrow 11 / 2^{-}$ & 1320 & $0.97(10)$ & $\begin{array}{c}0.99 \text { (10) ref. 8 } \\
>2.5 \text { ref. [7] }\end{array}$ & $83(4)$ & 81 & $0.246(12)$ \\
\hline $19 / 2^{-} \rightarrow 15 / 2^{-}$ & 1518 & $0.57(6)$ & 0.60 (6) ref. 8 & $75(3)$ & 65 & $0.221(8)$ \\
\hline \multirow{3}{*}{$23 / 2^{-} \rightarrow 19 / 2^{-}$} & \multirow{3}{*}{1770} & \multirow{3}{*}{$0.37(5)$} & 0.70 (10) ref. [7] & \multirow{3}{*}{$62(4)$} & \multirow{3}{*}{66} & \multirow{3}{*}{$0.185(12)$} \\
\hline & & & 0.35 (3) ref. 8 & & & \\
\hline & & & 0.50 (7) ref. [7] & & & \\
\hline
\end{tabular}


Fig. 1

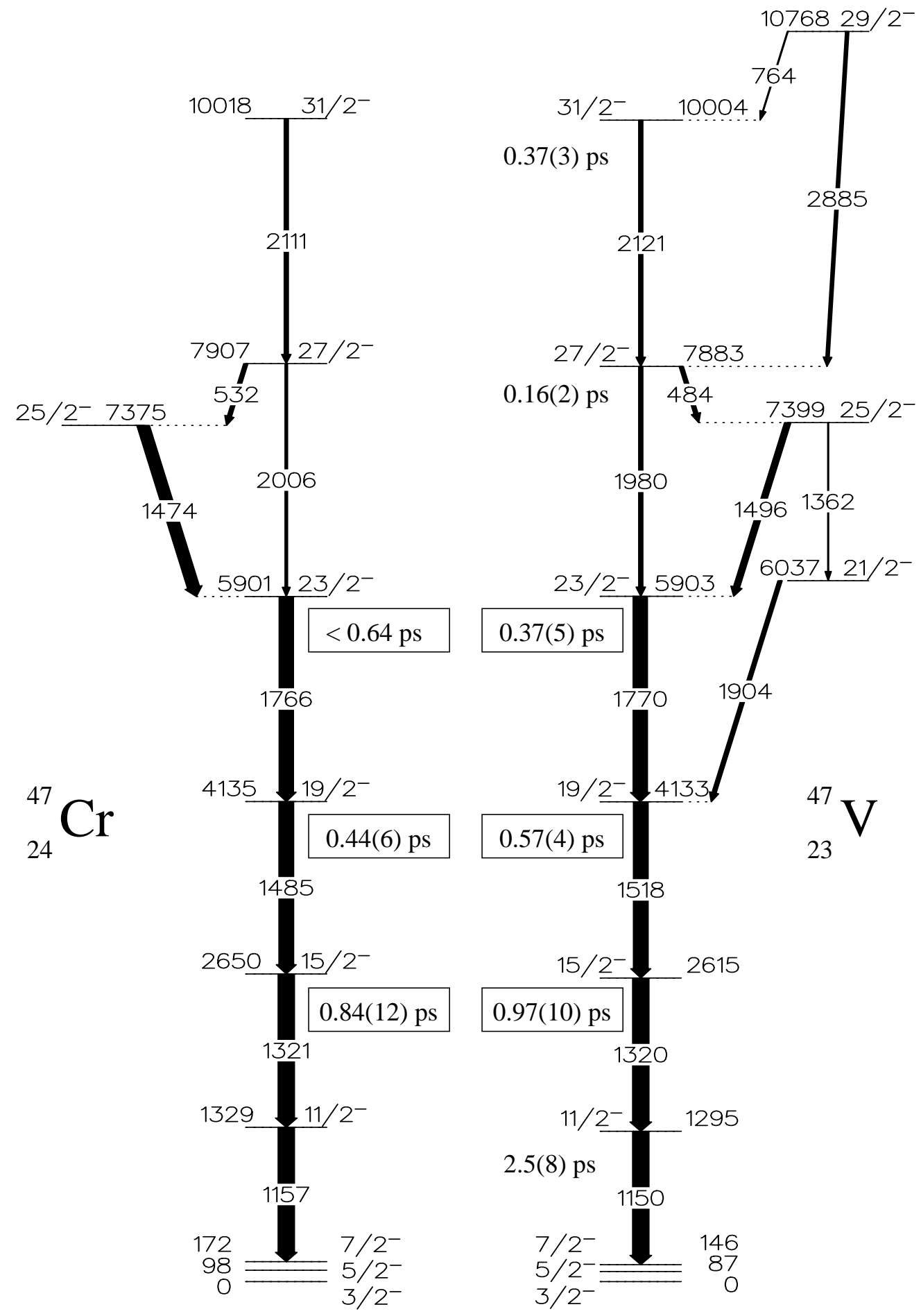


Fig. 2
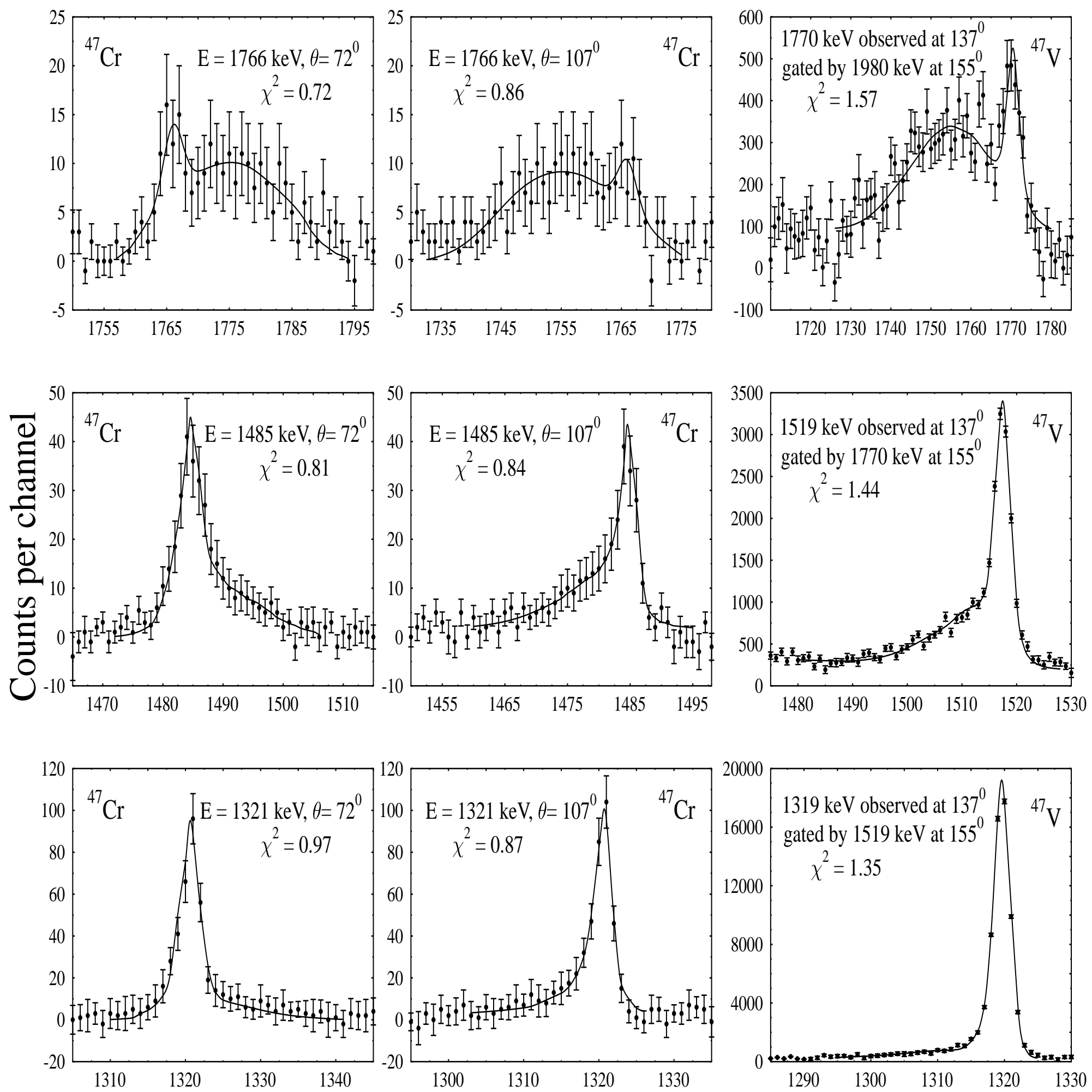

Gamma-ray energy (keV) 
Fig. 3

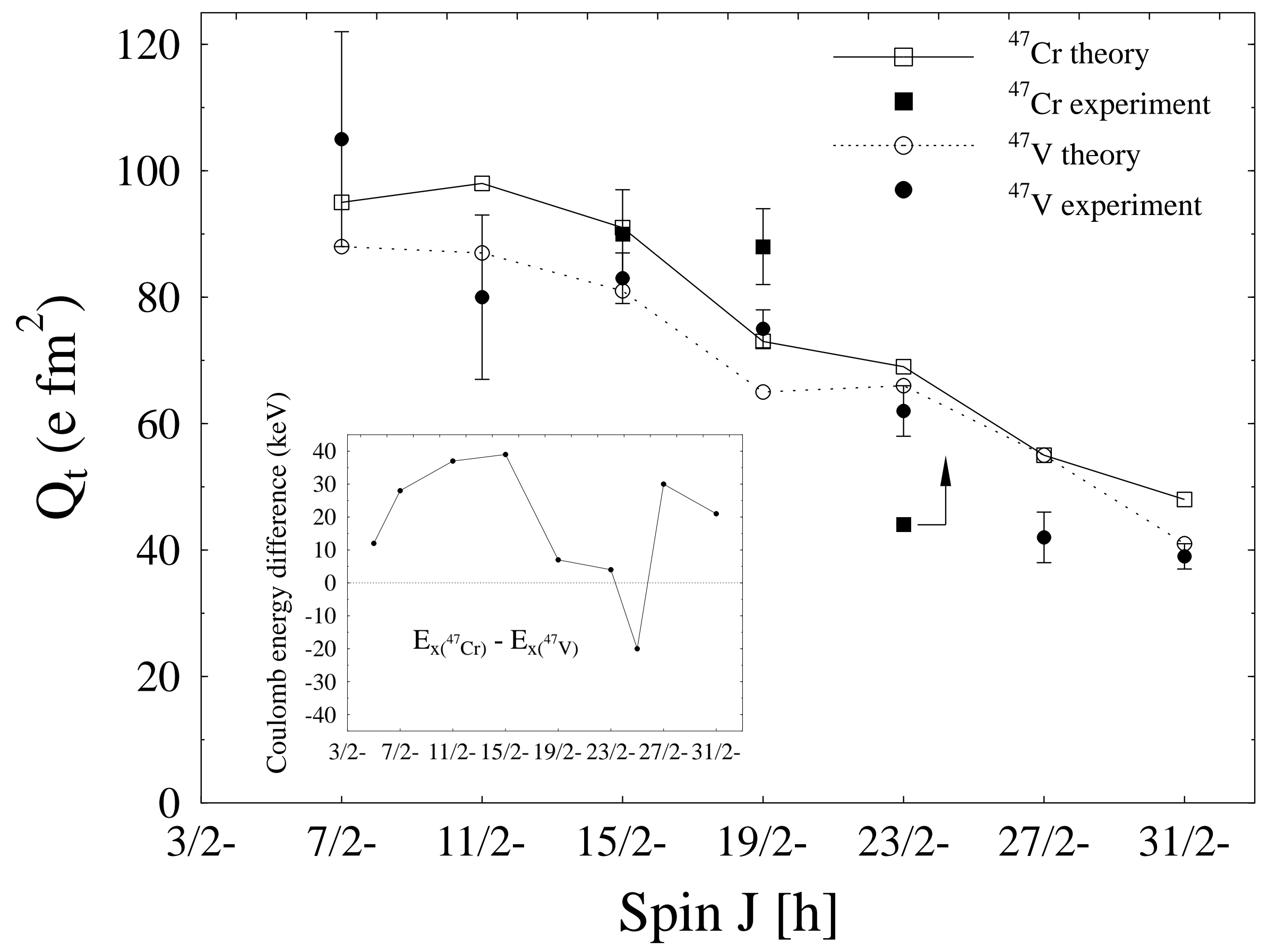

